\title{
Artificial Blood - A Game Changer for Future Medicine: Where are we Today?
}

\author{
Masoud Mozafari ${ }^{1^{*}}$, Arash Ramedani ${ }^{2}$ and Aboulfazl Yazdanpanah ${ }^{3}$ \\ ${ }^{1}$ Department of Nanotechnology and Advanced Materials Department, Bioengineering Research Group, Materials and Energy Research Center (MERC), P.O. Box \\ 14155-4777, Tehran, Iran
}

${ }^{2}$ Institute for Nanoscience \& Nanotechnology (INST), Sharif University of Technology, Tehran, Iran

${ }^{3}$ Biomaterials Group, Faculty of Biomedical Engineering (Center of Excellence), Amirkabir University of Technology, P.O. Box 15875-4413, Tehran, Iran

*Corresponding author: Masoud Mozafari, Department of Nanotechnology and Advanced Materials Department, Bioengineering Research Group, Materials and Energy Research Center (MERC), P.O. Box 14155-4777, Tehran, Iran, Tel: +98 912 6490679; Fax: +98 263 6280034x477; E-mail: mozafari.masoud@gmail.com

Received date: Sep 27, 2015, Accepted date: Sep 30, 2015, Publication date: Oct 05, 2015

Copyright: ( 2015 Mozafari M, et al. This is an open-access article distributed under the terms of the Creative Commons Attribution License, which permits unrestricted use, distribution, and reproduction in any medium, provided the original author and source are credited.

\section{Introduction}

Artificial blood is a new concept of biotechnology used to mimic and fulfil some functions of biological blood. Artificial blood aims to deliver an alternative to blood transfusion, which is transferring bloodbased products from a person to another one [1]. Hemoglobin is the main component of red blood cells, comprising about $33 \%$ of the cell mass. As an important type in this class, hemoglobin-based products are called hemoglobin-based oxygen carriers. However, pure hemoglobin separated from red cells cannot be used, since it causes renal toxicity. It can be treated to avoid this, but it still has incorrect oxygen transport characteristics when separated from red cells. Various other steps are needed to form hemoglobin into a useful and safe oxygen therapeutic agent. These may include cross-linking, polymerization, and encapsulation. These are needed because the red blood cell is not a simple container for haemoglobin, but a complex entity with many biomolecular features. The attempt to develop a feasible blood substitute goes back to more than seven decades ago. These efforts have essentially focused on the ability of red blood cells to carry oxygen. Hence, most of the products that are in the advancedphase of clinical trials are derivatives of hemoglobin. Currently, an increase in the number of surgeries and the risk of transmission of pathogens such as HIV are the most important motivations in the community for the development of synthetic substitutes for human blood. However, to date, no oxygen-carrying blood substitutes are approved for use by the US Food and Drug Administration (FDA). This fact not only shows the challenges in formulating effective blood substitutes but also indicates the essential needs and the immense potential for this field of study [2,3]. Today, this field is generally divided into four categories as recombinant hemoglobin, conjugated hemoglobin, polymerized hemoglobin, and liposome-encapsulated hemoglobin.

\section{Recombinant haemoglobin}

A large number of recombinant human hemoglobin can be expressed and purified from transgenic Escherichia coli. The physiological suitability of this material can be further enhanced using protein-engineering strategies to address specific efficacy and toxicity issues. Mutagenesis of hemoglobin can significantly adjust dioxygen affinity, reduce nitric oxide scavenging without compromising dioxygen binding, slow the rate of autoxidation and hemin loss, impede subunit dissociation, and diminish irreversible subunit denaturation. Recombinant hemoglobin production is potentially limitless but with spending a lot of money it is subjected to current good manufacturing practices. It has been frequently reported that acellular hemoglobin-based oxygen carriers have superior shelf-life compared to red blood cells. These carriers are outstanding alternatives when no other blood products are available or acceptable for a patient [4].

\section{Conjugated haemoglobin}

A class of synthetic and inert polymers, such as poly(ethylene) glycol (PEG) and Poly(L-lysine) (PLL), have been recently proposed to be able to progressively modify a wide range of drugs, genes, and proteins to increase their stability and functionality. For instance, recent studies reported that covalent attachments of poly(ethylene) glycol to the surfaces of acetylcholinesterase or interferon- $\beta-1 b$ have shown a meaningful increase in the circulatory residency times $[5,6]$. Similarly, conjugation of poly(ethylene) glycol with hemoglobin has been suggested to act a hemoglobin-based oxygen carrier.

\section{Polymerized haemoglobin}

Polyheme is a solution generated by extracting hemoglobin from red blood cells, and after being associated into tetramers the hemoglobin is mixed into an electrolyte solution [7]. This temporary oxygen-carrying blood substitute is in development for emergency cases where large volumes of blood are lost and fresh blood for transfusion is not available. In this strategy, the polymerization process is crucial due to the possibility of nephrotoxicity and liver failure from dissociated hemoglobin [8]. It has been reported that polyheme is compatible with all blood types and is stable for almost one year. This strategy offers practical solutions for the modification of human hemoglobin for applications in life-threatening situations.

\section{Liposome-encapsulated haemoglobin}

Another strategy is using an artificial neohemocyte as a capsule for hemoglobin to form hemoglobin-based oxygen carriers. The first generation of encapsulated hemoglobin was developed in the 1950s. This revolutionary finding had also technical limitations that slowed down further developments and improvements. Right after the HIV crisis public interests have come back to this innovation as a potentially viable strategy. Then, liposome-encapsulated hemoglobin has been found to be an effective oxygen carrier. It has been reported that this encapsulation strategy can increase plasma retention time. However, unwanted immune interactions may happen from the liposome capsule [9]. As a new strategy, microcapsulation of hemoglobin shed light over creating real artificial red cells including the essential enzymes that act for the reduction of ischemia reperfusion injury and solve problems associated with methaemoglobin formation [10]. The microcapsulation of hemoglobin production is still 
considered as a very challenging field of study due to the technical restrictions but there is hope for finding solutions for large-scale production of artificial blood.

There are also side effects associated with the use of different types of artificial bloods including hypertension, abdominal pain, diarrhea, jaundice, hemoglobinuria, oliguria, stroke, etc. Although these adverse effects have been mostly clinically asymptomatic, many clinical trials involving some of these agents have been discontinued. Despite many years of research in this area, finding an ideal artificial blood still continues to elude researchers. Unfortunately, initial attempts for introducing a clinically successful artificial blood have been always failed because of significant side effects. However, there is still hope to find the key, as continued researches has provided better understanding of the physiology of red blood cells and the interactions of these cells with their surrounding environment. The obtained knowledge helped us to produce the first generation of artificial blood while developing newer products that do not have significant vasoactive properties. Although there is still many unanswered questions, the proponents of producing artificial blood to enter routine clinical use is optimistic. Undoubtedly, extended research in all aspects of molecular biology need to be conducted to fully understand the interactive effects of artificial blood with the surrounding environment. Interdisciplinary researches and effective collaborations can potentially overcome the major issues related to large-scale production of blood substitutes in the near future (Figure 1).

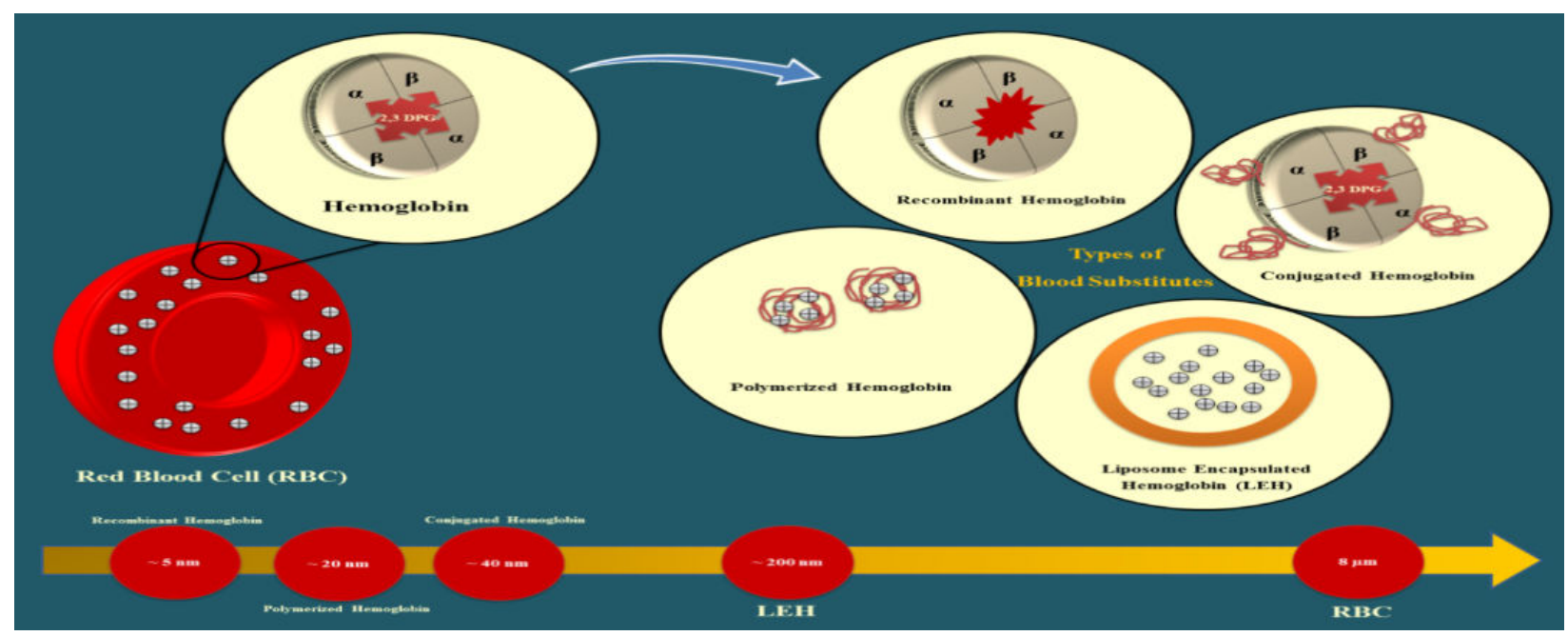

Figure 1: Development of Hemoglobin-based oxygen carriers as artificial blood. They are divided into four categories as: a) Recombinant hemoglobin: hemoglobin is genetically modified by Escherichia coli (size $5 \mathrm{~nm}$ ) b) Polymerized hemoglobin: hemoglobin is cross-linked by a polymer (size $20 \mathrm{~nm}$ ) c) ConjugatedI'm hemoglobin: hemoglobin is modified by a biocompatible polymer (size $40 \mathrm{~nm}$ ) d) Liposomeencapsulated hemoglobin: hemoglobin enclosed into a lipid membrane (size 200 nm). Hemoglobin-based oxygen carriers are smaller than red blood cells; therefore, it will better supply oxygen in the site of injury.

\section{References}

1. Spahn DR, Kocian R (2005) Artificial O2 carriers: status in 2005. Curr Pharm Des 11: 4099-4114.

2. Alayash AI (1999) Hemoglobin-based blood substitutes: oxygen carriers, pressor agents, or oxidants? Nat Biotechnol 17: 545-549.

3. Chen JY, Scerbo M, Kramer G (2009) A review of blood substitutes: examining the history, clinical trial results, and ethics of hemoglobin-based oxygen carriers. Clinics (Sao Paulo) 64: 803-813.

4. Varnado CL, Mollan TL, Birukou I, Smith BJ, Henderson DP, et al. (2013) Development of recombinant hemoglobin-based oxygen carriers. Antioxid Redox Signal 18: 2314-2328.

5. Kronman C, Cohen O, Raveh L, Mazor O, Ordentlich A, et al. (2006) Polyethylene-glycol conjugated recombinant human acetylcholinesterase serves as an efficacious bioscavenger against soman intoxication. Toxicology 233: 40-46.
6. Basu A, Yang K, Wang M, Liu S, Chintala R, et al. (2006) Structure-function engineering of interferon-beta-1b for improving stability, solubility, potency, immunogenicity, and pharmacokinetic properties by site-selective mono-PEGylation. Bioconjug Chem 17: 618-630.

7. Moore EE (2003) Blood substitutes: the future is now. J Am Coll Surg 196: $1-17$.

8. Winslow RM (2002) Blood substitutes. Curr Opin Hematol 9: 146-151.

9. Murayama C, Kawaguchi AT, Kamijo A, Naito K, Iwao K, et al. (2014) Liposome-encapsulated hemoglobin enhances chemotherapy to suppress metastasis in mice. Artif Organs 8: 656-661.

10. Kilian G, Milne P (2014) Optimization of selected liposome-encapsulated diketopiperazines. Journal of Drug Delivery Science and Technology 24: 143-147. 